\title{
Stability of Delayed Hopfield Neural Networks with Variable-Time Impulses
}

\author{
Yangjun Pei, ${ }^{1}$ Chao Liu, ${ }^{2}$ and Qi Han ${ }^{1}$ \\ ${ }^{1}$ School of Electrical and Information Engineering, Chongqing University of Science and Technology, Chongqing 401331, China \\ ${ }^{2}$ College of Computer Science and Engineering, Chongqing University of Technology, Chongqing 400050, China \\ Correspondence should be addressed to Chao Liu; xiuwenzheng2000@163.com
}

Received 3 April 2014; Accepted 22 July 2014; Published 1 September 2014

Academic Editor: Chuandong Li

Copyright (c) 2014 Yangjun Pei et al. This is an open access article distributed under the Creative Commons Attribution License, which permits unrestricted use, distribution, and reproduction in any medium, provided the original work is properly cited.

In this paper the globally exponential stability criteria of delayed Hopfield neural networks with variable-time impulses are established. The proposed criteria can also be applied in Hopfield neural networks with fixed-time impulses. A numerical example is presented to illustrate the effectiveness of our theoretical results.

\section{Introduction}

Hopfield neural networks [1], which were referred by Hopfield in 1984, have attracted many attentions of researchers and have been applied in many fields such as pattern recognition, associative memory, and combinatorial optimization. Stability, a crucial dynamic feature of Hopfield neural networks, has been intensively investigated over the past decades. Some significant sufficient results can be referred in [2-6].

It is well known that time delay is unavoidable due to finite switching speeds of the amplifiers and it may cause oscillations or instability of dynamic systems. The effects of time delay on the dynamical behavior of neural networks are nonnegligible. Some stability criteria for delayed Hopfield neural networks have been proposed in [7-10]. Meanwhile, impulsive phenomena exist in a wide variety of evolutionary processes, such as financial systems and nanoscale electronic circuits in which many state variables change instantaneously, in the form of impulses. On the other hand, impulsive control is also applied widely in many fields of information science, electronics, automated control systems, computer networking, artificial intelligence, robotics and telecommunications, and so forth. Neural networks may jump instantaneously because of environmental changes (such as external noise and disturbance). We may also introduce impulses deliberately to stabilize the oscillating and chaotic neural networks. Many researchers have investigated impulsive Hopfield neural networks and have obtained many interesting stability results [11-19].

However, up to now, the vast majority of stability results for impulsive Hopfield neural networks are focused on the case of fixed-time impulses. As we know, variabletime impulses arise naturally in biological and physiological systems. The primary difference between neural network with fixed-time impulses and neural network with variable-time impulses is the impulsive instant. In the neural network with fixed-time impulses, the impulsive instant is completely fixed and not about the state of system. But in neural network with variable-time impulses, the impulsive instant is not fixed and determined by state of system. In [20], we have focused on BAM neural networks with variable-time impulses and have obtained some crucial theoretical results. In [21], we have investigated the stabilizing effects of impulses for Hopfield neural networks and have shown that Hopfield neural networks with unstable continuous component may be still stable because of the stabilizing effects of impulses. In this paper, we focus on the destabilizing effects of Hopfield neural networks with variable-time impulses. It is shown that the impulsive Hopfield neural networks may preserve the global exponential stability of the impulse-free Hopfield neural networks even if the impulses have enlarging effects on the states of neurons. For this purpose, it is always assumed that the states of neurons enlarge at impulsive time. 
This paper is organized as follows. In the coming section we introduce some notations, definition, and lemmas. In Section 3 we consider the stability of Hopfield neural networks with time delays and variable-time impulses and establish stability criteria. In Section 4, one example is given to illustrate the effectiveness of our theoretical results.

\section{Preliminaries}

In this paper, we consider the following Hopfield neural networks with variable-time impulses:

$$
\begin{aligned}
\dot{\tilde{x}}(t) & =-A \tilde{x}(t)+B \tilde{f}(\tilde{x}(t-\tau))+\widetilde{I}, \quad t \neq \tilde{t}_{k}(x), \\
\Delta \widetilde{x}(t) & =\widetilde{J}_{k}\left(\tilde{x}\left(t^{-}\right)\right), \quad t=\widetilde{t}_{k}(x), \\
\tilde{x}\left(t_{0}+s\right) & =\widetilde{\phi}(s), \quad s \in[-\tau, 0],
\end{aligned}
$$

where $\tilde{x}=\left(\tilde{x}_{1}, \ldots, \tilde{x}_{n}\right)^{T}$ is the neuron state vector, $\tau>0$ is the transmission delay, $A=\operatorname{diag}\left(a_{1}, \ldots, a_{n}\right)$ with $a_{i}>0$, $i=1, \ldots, n, B=\left[b_{i j}\right]_{n \times n}$ is the delayed connection weight matrix, and $\tilde{f}(\cdot)=\left(\tilde{f}_{1}(\cdot), \ldots, \tilde{f}_{n}(\cdot)\right)^{T}$ is neuron activation function. $\widetilde{I}$ is the external input, $0 \leq t_{0}<\tilde{t}_{1}(\tilde{x})<\cdots<$ $\tilde{t}_{k}(\tilde{x})<\cdots, \tilde{t}_{k}(\tilde{x}) \rightarrow \infty$ as $k \rightarrow \infty, \tilde{x}\left(t^{+}\right)=\lim _{s \rightarrow t^{+}} \tilde{x}(s)$, and $\tilde{x}\left(t^{-}\right)=\lim _{s \rightarrow t^{-}} \tilde{x}(s)$. In this paper we always assume that $\tilde{x}(t)=\tilde{x}\left(t^{+}\right)$as $t=\widetilde{t}_{k}(\tilde{x})$. We further assume that

(A1) there is $l_{i}>0, i=1, \ldots, n$, such that $\left|\tilde{f}_{i}(\alpha)-\tilde{f}_{i}(\beta)\right| \leq$ $l_{i}|\alpha-\beta|$, for any $\alpha, \beta \in R$.

Throughout this paper, it is always assumed that there is at least one equilibrium point of (1). As usual, we shift an equilibrium point $x^{*}=\left(x_{1}^{*}, \ldots, x_{n}^{*}\right)^{T}$ to the origin by transformation $x=\tilde{x}-x^{*}$. Then system (1) can be rewritten as follows:

$$
\begin{gathered}
\dot{x}(t)=-A x(t)+B f(x(t-\tau)), \quad t \neq t_{k}(x), \\
\Delta x(t)=J_{k}\left(x\left(t^{-}\right)\right), \quad t=t_{k}(x), \\
x\left(t_{0}+s\right)=\phi(s), \quad s \in[-\tau, 0],
\end{gathered}
$$

where $f(x(t))=\tilde{f}\left(x(t)+x^{*}\right)-\tilde{f}\left(x^{*}\right), J_{k}\left(x\left(t^{-}\right)\right)=\widetilde{J}_{k}\left(x\left(t^{-}\right)+\right.$ $\left.x^{*}\right), t_{k}(x)=\widetilde{t}_{k}\left(x+x^{*}\right)$, and $\phi(s)=\widetilde{\phi}(s)-x^{*}$.

In the sequel, we introduce some notations, basic definition, and lemmas:

$$
\begin{gathered}
R_{+}=[0,+\infty) ; \quad S_{k}=\left\{(t, x) \in R_{+} \times R^{n}: t=t_{k}(x)\right\} \\
N_{k}=\left\{t \in R_{+}: \text {there is } x \in R^{n} \text { such that }(t, x) \in S_{k}\right\} \\
d_{k}=d\left(N_{k-1}, N_{k}\right)=\inf _{\substack{t \in N_{k} \\
t \in N_{k-1}}}|\bar{t}-t| ; \\
d_{k}^{*}=d^{*}\left(N_{k-1}, N_{k}\right)=\sup _{\substack{t \in N_{k} \\
t \in N_{k-1}}}|\bar{t}-t| ; \\
L=\operatorname{diag}\left(l_{1}, \ldots, l_{n}\right) ;
\end{gathered}
$$

$\lambda_{\max }(\cdot), \lambda_{\min }(\cdot)$ denote the maximum and the minimum eigenvalues of the corresponding matrix, respectively. $\|\cdot\|$ denotes the Euclidean norm of a vector or a square matrix.

$A>0$ denotes that $A$ is positive definite matrix.

$P C=P C\left(I, R^{n}\right)=\left\{\psi: I \rightarrow R^{n} \mid \psi\left(t^{+}\right)=\psi(t)\right.$ for $t \in I$, $\psi\left(t^{-}\right)$exists for $t \in I, \psi\left(t^{-}\right)=\psi(t)$ for all $t \in I$ but at most finite points $\}$, where $I$ is an interval:

$$
\|y\|_{\tau}=\sup _{\tau \leq s \leq 0}\|y(s)\| \quad \text { for } y \in P C\left([-\tau, 0], R^{n}\right) .
$$

Definition 1. The equilibrium point $x^{*}=\left(x_{1}^{*}, x_{2}^{*}, \ldots, x_{n}^{*}\right)^{T}$ of (1) is said to be globally exponentially stable if, for any solution $\tilde{x}\left(t, t_{0}, \tilde{\phi}\right)$ with the initial condition $\tilde{\phi} \in P C$, there are constant $\varepsilon>0$ and $M>1$ such that $\left\|\tilde{x}\left(t, t_{0}, \tilde{\phi}\right)-x^{*}\right\| \leq$ $M\left\|\widetilde{\phi}-x^{*}\right\|_{\tau} e^{-\varepsilon\left(t-t_{0}\right)}$.

Lemma 2 (Berman and Plemmons [22]). Let $A \in R^{n \times n}$; then

$$
\lambda_{\min }(A) x^{T} x \leq x^{T} A x \leq \lambda_{\max }(A) x^{T} x
$$

for any $x \in R^{n}$, if $A$ is a symmetric matrix.

Lemma 3. Consider the following differential inequality:

$$
\begin{gathered}
D^{+} V(t) \leq-\alpha V(t)+\beta V(t-\tau), \quad t \neq t_{k} \\
V(t) \leq c_{k} V\left(t^{-}\right), \quad t=t_{k}
\end{gathered}
$$

where $V(t) \geq 0, \alpha>\beta>0$, and $c_{k}>1, k=1,2, \ldots$ Denote $\delta^{*}=\sup _{k}\left\{t_{k+1}-t_{k}\right\}, \delta=\inf _{k}\left(t_{k+1}-t_{k}\right)$. For $d^{*} \geq \delta^{*}, 0<$ $d \leq \delta$, and $\theta>0$ such that $-\alpha+\beta e^{-\theta \tau} U=-\theta$, where $U=$ $\max _{k}\left\{c_{k}^{-1} \cdots c_{k+\underline{N}}^{-1}\right\}$ for $\underline{N}=\left\lfloor\tau / d^{*}\right\rfloor>0$ and $U=1$ for $\underline{N}=0$, one has

$$
V(t) \leq M \prod_{i=0}^{k-1} c_{i} \bar{V}\left(t_{0}\right) e^{-\theta\left(t-t_{0}\right)}, \quad t \in\left[t_{k-1}, t_{k}\right)
$$

where $c_{0}=1, \bar{V}\left(t_{0}\right)=\sup _{s \in[-\tau, 0]} V\left(t_{0}+s\right)$, and $M \geq 1$ is a constant.

Proof. See the Appendix section.

Remark 4. In Lemma 3, if $\tau<d^{*}$, we have $U=1$. Based on the proof, we know that $\theta=\varepsilon$ (please see the Appendix section for meaning of $\varepsilon$ ). If $\tau \geq d^{*}$, we obtain $U<1$. It is obvious to know that $\theta>\varepsilon$. Generally speaking, (7) is more valuable because the convergence rate in (7) is larger than that in (A.1) when $\tau \geq d^{*}$. Because $c_{k}>1$, we can use Lemma 3 to investigate the stability of impulsive differential systems in which the impulses are with destabilizing effects.

The solutions of system (1) may hit the same switching surface $S_{k}$ finite or infinite times causing "beating phenomenon" or "pulse phenomenon." In a similar way in [17], we can get the following lemma easily which guarantees that beating phenomenon does not exist. 
Lemma 5. Suppose that

(i) for any $k=1,2, \ldots \tilde{t}_{k}(x)$ is bounded;

(ii) for any $\left(t_{0}, \widetilde{\phi}\right)$ there is a solution of continuous subsystem of system (1) in $\left[t_{0},+\infty\right)$;

(iii) $\left(\partial \tilde{t}_{k}(\tilde{x}) / \partial \tilde{x}\right)(-A \tilde{x}+B \tilde{f}(\tilde{x}(t-\tau))+I)<1$;

(iv) $\tilde{t}_{k}(\tilde{x}) \geq \tilde{t}_{k}\left(\tilde{x}+\widetilde{J}_{k}(\tilde{x})\right)$, and $\tilde{t}_{k+1}\left(\tilde{x}+\widetilde{J}_{k}(\tilde{x})\right)>\tilde{t}_{k}(\tilde{x})$.

Then there is solution of system (1) in $\left[t_{0},+\infty\right)$, and it hits each switching surface $S_{k}: t=\widetilde{t}_{k}(\tilde{x})$ exactly once in turn.

From Theorem 1.1.4 in [23], we know that there exists a unique solution of system (1) without impulses in our paper on $\left[t_{0},+\infty\right)$, if $\|f(x)-f(y)\| \leq K\|x-y\|$, which yields that there exists a unique solution for any $\sum_{k}=\{(t, x)$ : $\left.t_{k-1}(x) \leq t<t_{k}(x)\right\}$ and any initial condition. Therefore, by mathematical induction, we know the global uniqueness and existence of solution. From now on we always assume that there exists a unique solution of system (1) $\tilde{x}(t)=\tilde{x}\left(t, t_{0}, \widetilde{\phi}\right)$ satisfying the conditions of Lemma 5; namely, it hits each switching surface $S_{i}, i \geq k$, only once [11]. In addition, we also always assume that $\left\{t_{i}\right\}_{i=1}^{\infty}$ are the moments that integral curve $\left(t, \tilde{x}\left(t, t_{0}, \widetilde{\phi}\right)\right)$ hits each switching surface $\left\{S_{k_{i}}\right\}_{i=1}^{\infty}$ in turn; namely, $t_{i}=\tilde{t}_{k_{i}}\left(x\left(t_{i}\right)\right)$ and $t_{i}<t_{i+1}$.

\section{Main Results}

In this section, we establish some sufficient criteria for the exponential stability of system (1).

Theorem 6. Assume that, in addition to condition (A1), the following conditions are satisfied:

(A2) $\widetilde{J}_{k}(\tilde{x}(t))=S_{k}\left(\tilde{x}(t)-x^{*}\right)$ with $S_{k}=\operatorname{diag}\left(s_{1 k}, s_{2 k}, \ldots\right.$, $\left.s_{n k}\right)$

(A3) $d=\inf _{k}\left\{d_{k}\right\}>0, d^{*}=\sup _{k}\left\{d_{k}^{*}\right\}<\infty$;

(A4) there are a symmetric positive definite matrix $P$, constants $\mu>0, q>0, c_{k}>0, \varepsilon>0$, and $\alpha>\beta$ such that

$$
\begin{gathered}
-A^{T} P-P A+\mu^{-1} P B B^{T} P \leq-\alpha P, \\
\mu L^{T} L \leq \beta P \\
P+S_{k}^{T} P+P S_{k}+S_{k}^{T} P S_{k} \leq c_{k} P,
\end{gathered}
$$

$c_{k}<e^{\varepsilon d}$, and $-\alpha+\beta e^{\varepsilon \tau} U=-\varepsilon$, where $U=\max _{k}\left\{c_{k}^{-1} \cdots c_{k+\underline{N}}^{-1}\right\}$ for $N=\left\lfloor\tau / d^{*}\right\rfloor>0$ and $U=1$ for $N=0$.

Then the equilibrium point $x^{*}$ of system (1) is globally exponentially stable.

Proof. Based on (A2), we know that $J_{k}\left(x\left(t^{-}\right)\right)=S_{k} x\left(t^{-}\right)$. We choose the Lyapunov function of system (2) as follows:

$$
V(t, x(t))=x^{T}(t) P x(t) .
$$

Let $V(t)=V(t, x(t))$ briefly. When $t \neq t_{i}$, we have

$$
\begin{aligned}
D^{+} V(t)= & \dot{x}^{T}(t) P x(t)+x^{T}(t) P x(t) \\
= & (-A x(t)+B f(x(t-\tau)))^{T} P x(t) \\
& +x^{T}(t) P(-A x(t)+B f(x(t-\tau))) \\
\leq & x^{T}(t)\left(-A^{T} P-P A\right) x(t) \\
& +\mu^{-1} x^{T}(t) P B B^{T} P x(t)+\mu f^{T}(x(t)) f(x(t)) \\
\leq & x^{T}(t)\left(-A^{T} P-P A+\mu^{-1} P B B^{T}\right) x(t) \\
& +\mu^{-1} x^{T}(t-\tau) L^{T} L x(t-\tau) \\
\leq & -\alpha V(t)+\beta V(t-\tau) .
\end{aligned}
$$

When $t=t_{i}$, we have

$$
\begin{aligned}
V\left(t_{i}\right) & =x^{T}\left(t_{i}\right) P x\left(t_{i}\right) \\
& =\left(x\left(t_{i}^{-}\right)+S_{k_{i}} x\left(t_{i}^{-}\right)\right)^{T} P\left(x\left(t_{i}^{-}\right)+S_{k_{i}} x\left(t_{i}^{-}\right)\right) \\
& =x^{T}\left(t_{i}^{-}\right)\left(P+S_{k_{i}}^{T} P+P S_{k_{i}}+S_{k_{i}}^{T} P S_{k_{i}}\right) x\left(t_{i}^{-}\right) \\
& \leq c_{k_{i}} V\left(t_{i}^{-}\right) .
\end{aligned}
$$

Therefore, we have

$$
\begin{gathered}
D^{+} V(t) \leq-\alpha V(t)+\beta V(t-\tau), \quad t \neq t_{i}, \\
V\left(t_{i}\right) \leq c_{k_{i}} V\left(t_{i}^{-}\right), \quad i=1,2, \ldots
\end{gathered}
$$

On the basis of (7) and Lemma 3, we have

$$
V(t) \leq M \prod_{l=1}^{i} c_{k_{l}} \bar{V}\left(t_{0}\right) e^{-\varepsilon\left(t-t_{0}\right)}, \quad t \in\left[t_{i}, t_{i+1}\right) .
$$

From condition (A4), we know that there is $0<\zeta<\varepsilon$ such that $c_{l} \leq e^{(\varepsilon-\zeta) d} \leq e^{(\varepsilon-\zeta)\left(t_{l+1}-t_{l}\right)}, l=1,2, \ldots$. Therefore, we have

$$
\begin{aligned}
V(t) & \leq M \prod_{l=1}^{i} e^{(\varepsilon-\zeta)\left(t_{l+1}-t_{l}\right)} e^{-\varepsilon\left(t-t_{0}\right)} \\
& =M e^{-\zeta\left(t-t_{0}\right)} e^{(\varepsilon-\zeta)\left(t_{i+1}-t+t_{0}-t_{1}\right)} \\
& \leq M e^{-\zeta\left(t-t_{0}\right)} e^{(\varepsilon-\zeta) d^{*}} .
\end{aligned}
$$

Denote $\bar{M}=e^{(\varepsilon+\zeta) d^{*}} ;$ then we have

$$
V(t) \leq M \bar{M} \bar{V}\left(t_{0}\right) e^{-\zeta\left(t-t_{0}\right)} .
$$

Based on (9) and Lemma 2, we obtain that

$$
\|x(t)\| \leq \sqrt{\frac{M \bar{M} \lambda_{\max }(P)}{\lambda_{\min }(P)}}\|\widetilde{\phi}\|_{\tau} e^{-(\zeta / 2)\left(t-t_{0}\right)} .
$$


By virtue of $x(t)=\widetilde{x}(t)-x^{*}$, we have

$$
\left\|\widetilde{x}(t)-x^{*}\right\| \leq \sqrt{\frac{M \bar{M} \lambda_{\max }(P)}{\lambda_{\min }(P)}}\left\|\widetilde{\phi}-x^{*}\right\|_{\tau} e^{-(\zeta / 2)\left(t-t_{0}\right)}
$$

which yields that the equilibrium point $x^{*}$ of system (1) is globally exponentially stable.

Remark 7. Because $\alpha>\beta$, we know that impulse-free neural network is stable. The impulses may be of destabilizing effects due to $c_{k}>1$. It is shown that impulsive Hopfield neural networks will preserve the global exponential stability of the impulse-free Hopfield neural networks even if the impulses have enlarging effects on the states of neurons.

As mentioned in [17], the impulsive differential systems in which impulses occur in fixed time can be viewed as particular impulsive differential systems with variable-time impulses. Therefore, based on Theorem 6, we can obtain the stability criterion for the following format of Hopfield neural networks:

$$
\begin{gathered}
\dot{\tilde{x}}(t)=-A \widetilde{x}(t)+B \tilde{f}(\tilde{x}(t-\tau))+\widetilde{I}, \quad t \neq \widetilde{t}_{k}, \\
\Delta \widetilde{x}(t)=\widetilde{J}_{k}\left(\widetilde{x}\left(t^{-}\right)\right), \quad t=\widetilde{t}_{k}, \\
\tilde{x}\left(t_{0}+s\right)=\widetilde{\phi}(s), \quad s \in[-\tau, 0] .
\end{gathered}
$$

Theorem 8. Assume that (A1), (A2) hold, and

(A5) $\delta=\inf _{k}\left\{\delta_{k}\right\}>0, \delta^{*}=\sup _{k}\left\{\delta_{k}^{*}\right\}<\infty$;

(A6) there are a symmetric positive definite matrix $P$, constants $\mu>0, q>0, c_{k}>0, \varepsilon>0$, and $\alpha>\beta$ such that

$$
\begin{gathered}
-A^{T} P-P A+\mu^{-1} P B B^{T} P \leq-\alpha P, \\
\mu L^{T} L \leq \beta P, \\
P+S_{k}^{T} P+P S_{k}+S_{k}^{T} P S_{k} \leq c_{k} P,
\end{gathered}
$$

$c_{k}<e^{\varepsilon d}$, and $-\alpha+\beta e^{\varepsilon \tau} U=-\varepsilon$, where $U=\max _{k}\left\{c_{k}^{-1} \cdots c_{k+\underline{N}}^{-1}\right\}$ for $\underline{N}=\left\lfloor\tau / \delta^{*}\right\rfloor>0$ and $U=1$ for $\underline{N}=0$.

Then the equilibrium point $x^{*}$ of system (18) is globally exponentially stable.

\section{Numeric Example}

In this section, we consider one example to illustrate the effectiveness of theoretical results.
Example 1. Consider the following system:

$$
\begin{gathered}
\dot{\tilde{x}}_{1}(t)=-\widetilde{x}_{1}(t)+0.1 \sin \left(\widetilde{x}_{1}(t-0.8)\right) \\
+0.4 \sin \left(\widetilde{x}_{2}(t-0.8)\right)+\pi, \\
\dot{\tilde{x}}_{2}(t)=-\dot{\tilde{x}}_{2}(t)+0.4 \sin \left(\widetilde{x}_{1}(t-0.8)\right) \\
+0.1 \sin \left(\widetilde{x}_{2}(t-0.8)\right)+\pi, \quad t \neq \widetilde{t}_{k}(\widetilde{x}), \\
\Delta \widetilde{x}_{1}(t)=-2.2\left(\widetilde{x}_{1}(t)-\pi\right), \\
\Delta \widetilde{x}_{2}(t)=-2.2\left(\widetilde{x}_{2}(t)-\pi\right), \quad t=\widetilde{t}_{k}(\widetilde{x}),
\end{gathered}
$$

where $\tilde{t}_{k}(\tilde{x})=-\left(2 / 5 \pi^{2}\right) \arctan ^{2}\left(\tilde{x}_{1}+\tilde{x}_{2}-2 \pi\right)+0.7 k$. It is easy to obtain that $A=\operatorname{diag}(1,1), B=\left[\begin{array}{ll}0.1 & 0.4 \\ 0.4 & 0.1\end{array}\right], S_{k}=$ $\operatorname{diag}(-2.2,-2.2), \tau=0.8, d=0.6, d^{*}=0.8, L=E$, and $x^{*}=(\pi, \pi)^{T}$.

Now we verify that there is no beating phenomenon in system (20).

(1) It is obvious that, for $k=1,2, \ldots, \widetilde{t}_{k}(x)=$ $-\left(2 / 5 \pi^{2}\right) \arctan ^{2}\left(\widetilde{x}_{1}+\widetilde{x}_{2}-2 \pi\right)+0.7 k$ is bounded.

(2) Based on [24], it is easy to predicate the existence of solutions for system (20).

(3) Let $y=\widetilde{x}_{1}+\widetilde{x}_{2}-2 \pi, F_{1}=\sin \left(\widetilde{x}_{1}(t-0.8)\right)$, and $F_{2}=$ $\sin \left(\widetilde{x}_{2}(t-0.8)\right)$. We have

$$
\begin{aligned}
& \frac{\partial \widetilde{t}_{k}(\tilde{x})}{\partial \widetilde{x}}(-A \widetilde{x}+B \tilde{f}(\tilde{x}(t-\tau))+I) \\
& \quad=-\frac{4}{5 \pi^{2}} \frac{\arctan (y)}{1+y^{2}}\left(-y+0.5 F_{1}+0.5 F_{2}\right) \\
& \quad \leq \frac{4}{5 \pi^{2}} \frac{|y|}{1+y^{2}}\left(|y|+0.5\left|F_{1}\right|+0.5\left|F_{2}\right|\right) \\
& \quad \leq \frac{4}{5 \pi^{2}}\left(\frac{y^{2}}{1+y^{2}}+0.5\left(\frac{\left|F_{1}\right|+\left|F_{2}\right|}{1+y^{2}}\right)\right) \leq \frac{8}{5 \pi^{2}}<1 .
\end{aligned}
$$

(4) It is easy to see that $\widetilde{t}_{k}(\widetilde{x}) \geq t_{k}\left(\widetilde{x}+\widetilde{J}_{k}(\widetilde{x})\right)$ and $\widetilde{t}_{k+1}(\widetilde{x}+$ $\left.\tilde{J}_{k}(\widetilde{x})\right)>\tilde{t}_{k}(\widetilde{x})$.

Therefore, all the conditions of Lemma 5 are satisfied; that is to say, there is no beating phenomenon in system (20).

For convenience, choose $P=E, \mu=1, \alpha=1.7, \beta=1$, and $c_{k}=1.44$. It is easy to verify that $\varepsilon=0.792$ and all the conditions of Theorem 6 are satisfied. Therefore, system (20) is globally exponentially stable, although the impulses are of destabilizing effects, as shown in Figure 1.

\section{Appendix}

\section{Proof of Lemma 3}

Let $\varepsilon$ be the largest positive solution satisfying the inequality $-\alpha+\beta e^{\varepsilon \varsigma} \leq-\varepsilon$. We claim that

$$
V(t) \leq \prod_{i=0}^{k-1} c_{i} \bar{V}\left(t_{0}\right) e^{-\varepsilon\left(t-t_{0}\right)},
$$

where $t \in\left[t_{k-1}, t_{k}\right), k=1,2, \ldots$ 


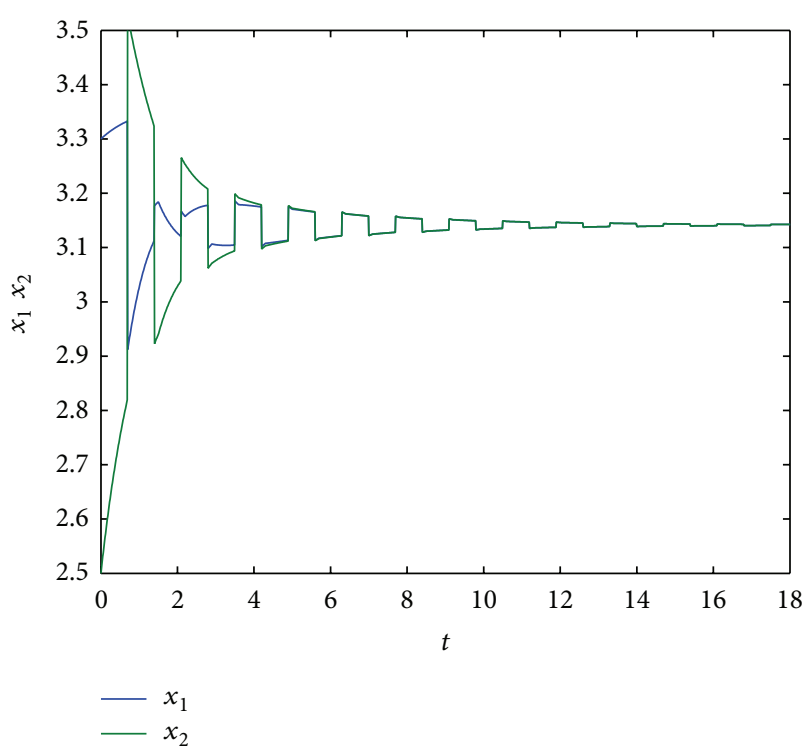

FIGURE 1: The time response curves of system (20).

First, for $t \in\left[t_{0}-\tau, t_{0}\right]$, we have $V(t) \leq \bar{V}\left(t_{0}\right) \leq$ $\bar{V}\left(t_{0}\right) e^{-\varepsilon\left(t-t_{0}\right)}$. Particularly, $V\left(t_{0}\right) \leq \bar{V}\left(t_{0}\right) e^{-\varepsilon\left(t_{0}-t_{0}\right)}=$ $c_{0} \bar{V}\left(t_{0}\right) e^{-\varepsilon\left(t_{0}-t_{0}\right)}$. Now we show that (A.1) holds for $k=1$; namely,

$$
V(t) \leq c_{0} \bar{V}\left(t_{0}\right) e^{-\varepsilon\left(t-t_{0}\right)}, \quad t \in\left[t_{0}, t_{1}\right) .
$$

If (A.2) does not hold, there is $t^{*} \in\left[t_{0}, t_{1}\right)$ such that

$$
\begin{gathered}
V\left(t^{*}\right)=c_{0} \bar{V}\left(t_{0}\right) e^{-\varepsilon\left(t^{*}-t_{0}\right)}, \\
D^{+} V\left(t^{*}\right)>-\varepsilon c_{0} \bar{V}\left(t_{0}\right) e^{-\varepsilon\left(t^{*}-t_{0}\right)} .
\end{gathered}
$$

However, based on (6), we have

$$
\begin{aligned}
D^{+} V\left(t^{*}\right) & \leq-\alpha V\left(t^{*}\right)+\beta V\left(t^{*}-\tau\right) \\
& \leq-\alpha c_{0} \bar{V}\left(t_{0}\right) e^{-\varepsilon\left(t^{*}-t_{0}\right)}+\beta c_{0} \bar{V}\left(t_{0}\right) e^{-\varepsilon\left(t^{*}-\tau-t_{0}\right)} \\
& =\left(-\alpha+\beta e^{\varepsilon \tau}\right) c_{0} \bar{V}\left(t_{0}\right) e^{-\varepsilon\left(t^{*}-t_{0}\right)} \\
& \leq-\varepsilon c_{0} \bar{V}\left(t_{0}\right) e^{-\varepsilon\left(t^{*}-t_{0}\right)},
\end{aligned}
$$

which contradicts (A.3).

Now suppose that, for $k=\bar{k}, \bar{k} \geq 1$, (A.1) holds. Namely, $V(t) \leq \prod_{i=0}^{\bar{k}-1} c_{i} \bar{V}\left(t_{0}\right) e^{-\varepsilon\left(t-t_{0}\right)}, t \in\left[t_{\bar{k}-1}, t_{\bar{k}}\right)$. We prove that (A.1) is also satisfied for $k=\bar{k}+1$. From (6) we can obtain $V\left(t_{\bar{k}}\right)=$ $\prod_{i=0}^{\bar{k}} c_{i} \bar{V}\left(t_{0}\right) e^{-\varepsilon\left(t_{\bar{k}}-t_{0}\right)}$. If this claim is not true, there is $t^{* *} \epsilon$ $\left[t_{\bar{k}}, t_{\bar{k}+1}\right)$ such that

$$
\begin{gathered}
V\left(t^{* *}\right)=\prod_{i=0}^{\bar{k}} c_{i} \bar{V}\left(t_{0}\right) e^{-\varepsilon\left(t^{* *}-t_{0}\right)}, \\
D^{+} V\left(t^{* *}\right)>-\varepsilon \prod_{i=0}^{\bar{k}} c_{i} \bar{V}\left(t_{0}\right) e^{-\varepsilon\left(t^{* *}-t_{0}\right)} .
\end{gathered}
$$

There is a nonnegative integer $h(0 \leq h \leq \bar{k}+1)$ such that $t^{* *}-\tau \in\left[t_{h-1}, t_{h}\right)\left(\right.$ if $\left.h=0, t_{h-1}=t_{0}-\tau\right)$. Then we have

$$
V\left(t^{* *}-\tau\right) \leq \prod_{i=0}^{h-1} c_{i} \bar{V}\left(t_{0}\right) e^{-\varepsilon\left(t^{* *}-\tau-t_{0}\right)} .
$$

By virtue of (6), we obtain

$$
\begin{aligned}
D^{+} V\left(t^{* *}\right) \leq & -\alpha V\left(t^{* *}\right)+\beta V\left(t^{* *}-\tau\right) \\
\leq & -\alpha \prod_{i=0}^{\bar{k}} c_{i} \bar{V}\left(t_{0}\right) e^{-\varepsilon\left(t^{* *}-t_{0}\right)} \\
& +\beta \prod_{i=0}^{h-1} c_{i} \bar{V}\left(t_{0}\right) e^{-\varepsilon\left(t^{* *}-\tau-t_{0}\right)} \\
= & \prod_{i=0}^{\bar{k}} c_{i} \bar{V}\left(t_{0}\right) e^{-\varepsilon\left(t^{* *}-t_{0}\right)}\left(-\alpha+\beta \prod_{i=h}^{\bar{k}} c_{i}^{-1} e^{\varepsilon \tau}\right) \\
\leq & \prod_{i=0}^{\bar{k}} c_{i} \bar{V}\left(t_{0}\right) e^{-\varepsilon\left(t^{* *}-t_{0}\right)}\left(-\alpha+\beta e^{\varepsilon \tau}\right) \\
\leq & -\varepsilon \prod_{i=0}^{\bar{k}} c_{i} \bar{V}\left(t_{0}\right) e^{-\varepsilon\left(t^{* *}-t_{0}\right)},
\end{aligned}
$$

which contradicts (A.5), which yields that (A.1) holds for $k=$ $\bar{k}+1$.

We can find $k^{*} \geq 1$ such that $t_{k^{*}}-\tau \geq t_{0}$. For any $t \in$ $\left[t_{k-1}, t_{k}\right), k \leq k^{*}$, from (A.1) we have

$$
\begin{aligned}
V(t) & \leq \prod_{i=0}^{k-1} c_{i} \bar{V}\left(t_{0}\right) e^{-\varepsilon\left(t-t_{0}\right)} \\
& \leq \prod_{i=0}^{k-1} c_{i} \bar{V}\left(t_{0}\right) e^{-\varepsilon\left(t-t_{0}\right)} e^{(\theta-\varepsilon)\left(t-t_{0}\right)} \\
& \leq \prod_{i=0}^{k-1} c_{i} \bar{V}\left(t_{0}\right) e^{-\theta\left(t-t_{0}\right)} e^{(\theta-\varepsilon)\left(k^{*} d^{*}+t_{1}-t_{0}\right)} \\
& =M \prod_{i=0}^{k-1} c_{i} \bar{V}\left(t_{0}\right) e^{-\theta\left(t-t_{0}\right)}
\end{aligned}
$$

where $M=e^{(\theta-\varepsilon)\left(k^{*} d^{*}+t_{1}-t_{0}\right)}$. That is to say, (7) holds for any $k \leq k^{*}$.

Now we show that (7) holds for $k>k^{*}$. For $k=k^{*}+1$, if (7) does not hold, there is $t^{* * *} \in\left[t_{k^{*}}, t_{k^{*}+1}\right)$ such that

$$
\begin{gathered}
V\left(t^{* * *}\right)=M \prod_{i=0}^{k^{*}} c_{i} \bar{V}\left(t_{0}\right) e^{-\theta\left(t^{* * *}-t_{0}\right)}, \\
D^{+} V\left(t^{* * *}\right)>-\theta M \prod_{i=0}^{k^{*}} c_{i} \bar{V}\left(t_{0}\right) e^{-\theta\left(t^{* * *}-t_{0}\right)} .
\end{gathered}
$$


Note that there is a positive integer $h^{*}\left(1 \leq h^{*} \leq k^{*}+1\right)$ such that $t^{* * *}-\tau \in\left[t_{h^{*}-1}, t_{h^{*}}\right)$. Then we have

$$
\begin{aligned}
D^{+} V\left(t^{* * *}\right) & \\
\leq & -\alpha V\left(t^{* * *}\right)+\beta V\left(t^{* * *}-\tau\right) \\
\leq & -\alpha M \prod_{i=0}^{k^{*}} c_{i} \bar{V}\left(t_{0}\right) e^{-\theta\left(t^{* * *}-t_{0}\right)} \\
& +\beta M \prod_{i=0}^{h^{*}-1} c_{i} \bar{V}\left(t_{0}\right) e^{-\theta\left(t^{* * *}-\tau-t_{0}\right)} \\
\leq & M \prod_{i=0}^{k^{*}} c_{i} \bar{V}\left(t_{0}\right) e^{-\theta\left(t^{* * *}-t_{0}\right)}\left(-\alpha+\beta \prod_{i=h^{*}} c_{i}^{-1} e^{\theta \tau}\right) \\
\leq & M \prod_{i=0}^{k^{*}} c_{i} \bar{V}\left(t_{0}\right) e^{-\theta\left(t^{* * *}-t_{0}\right)}\left(-\alpha+\beta e^{\theta \tau} U\right) \\
\leq & -\theta M \prod_{i=0}^{k^{*}} c_{i} \bar{V}\left(t_{0}\right) e^{-\theta\left(t^{* * *}-t_{0}\right)},
\end{aligned}
$$

which contradicts (A.9). Therefore (7) holds for $k=k^{*}+1$.

By mathematical induction, it is easy to illustrate that (7) holds for $k>k^{*}+1$. The proof is complete.

\section{Conflict of Interests}

The authors declare that there is no conflict of interests regarding the publication of this paper.

\section{Acknowledgments}

This research is funded by the Research Foundation of The Natural Foundation of Chongqing City (cstc2014jcyjA40024, cstc2012jjA1459), Teaching \& Research Program of Chongqing Education Committee (KJ1401307, KJ131401), and Research Project of Chongqing University of Science and Technology (CK2013B15).

\section{References}

[1] J. J. Hopfield, "Neurons with graded response have collective computational properties like those of two-state neurons," Proceedings of the National Academy of Sciences of the United States of America, vol. 81, no. 10, pp. 3088-3092, 1984.

[2] Z. H. Guan, G. R. Chen, and Y. Qin, "On equilibria, stability, and instability of Hopfield neural networks," IEEE Transactions on Neural Networks, vol. 11, no. 2, pp. 534-540, 2000.

[3] J. C. Juang, "Stability analysis of Hopfield-type neural networks," IEEE Transactions on Neural Networks, vol. 10, no. 6, pp. 13661374, 1999.

[4] D. Lee, "New stability conditions for Hopfield networks in partial simultaneous update mode," IEEE Transactions on Neural Networks, vol. 10, no. 4, pp. 975-978, 1999.

[5] X. B. Liang, "Equivalence between local exponential stability of the unique equilibrium point and global stability for Hopfieldtype neural networks with two neurons," IEEE Transactions on Neural Networks, vol. 11, no. 5, pp. 1194-1196, 2000.
[6] H. Yang and T. S. Dillon, "Exponential stability and oscillation of Hopfield graded response neural network," IEEE Transactions on Neural Networks, vol. 5, no. 5, pp. 719-729, 1994.

[7] T. Chen, "Global exponential stability of delayed Hopfield neural networks," Neural Networks, vol. 14, no. 8, pp. 977-980, 2001.

[8] K. Gopalsamy and X. Z. He, "Stability in asymmetric Hopfield nets with transmission delays," Physica D: Nonlinear Phenomena, vol. 76, no. 4, pp. 344-358, 1994.

[9] P. van den Driessche and X. F. Zou, "Global attractivity in delayed Hopfield neural network models," SIAM Journal on Applied Mathematics, vol. 58, no. 6, pp. 1878-1890, 1998.

[10] D. Xu, H. Zhao, and H. Zhu, "Global dynamics of Hopfield neural networks involving variable delays," Computers \& Mathematics with Applications, vol. 42, no. 1-2, pp. 39-45, 2001.

[11] H. Akça, R. Alassar, V. Covachev, Z. Covacheva, and E. Al-Zahrani, "Continuous-time additive Hopfield-type neural networks with impulses," Journal of Mathematical Analysis and Applications, vol. 290, no. 2, pp. 436-451, 2004.

[12] Z. H. Guan and G. Chen, "On delayed impulsive Hopfield neural networks," Neural Networks, vol. 12, no. 2, pp. 273-280, 1999.

[13] Z. T. Huang, Q. G. Yang, and X. S. Luo, "Exponential stability of impulsive neural networks with time-varying delays," Chaos, Solitons and Fractals, vol. 35, no. 4, pp. 770-780, 2008.

[14] S. Long and D. Xu, "Delay-dependent stability analysis for impulsive neural networks with time varying delays," Neurocomputing, vol. 71, no. 7-9, pp. 1705-1713, 2008.

[15] X. F. Yang, X. Liao, D. J. Evans, and Y. Tang, "Existence and stability of periodic solution in impulsive Hopfield neural networks with finite distributed delays," Physics Letters A, vol. 343, no. 1-3, pp. 108-116, 2005.

[16] C. J. Li, C. D. Li, and T. W. Huang, "Exponential stability of impulsive high-order Hopfield-type neural networks with delays and reaction-diffusion," International Journal of Computer Mathematics, vol. 88, no. 15, pp. 3150-3162, 2011.

[17] C. Li, C. Li, X. Liao, and T. Huang, "Impulsive effects on stability of high-order BAM neural networks with time delays," Neurocomputing, vol. 74, no. 10, pp. 1541-1550, 2011.

[18] C. J. Li, W. W. Yu, and T. W. Huang, "Impulsive synchronization schemes of stochastic complex networks with switching topology: average time approach," Neural Networks, vol. 54, pp. 8594, 2014.

[19] Q. Han, X. Liao, and C. Li, "Analysis of associative memories based on stability of cellular neural networks with time delay," Neural Computing and Applications, vol. 23, no. 1, pp. 237-244, 2013.

[20] C. Liu, C. Li, and X. Liao, "Variable-time impulses in BAM neural networks with delays," Neurocomputing, vol. 74, no. 17, pp. 3286-3295, 2011.

[21] C. Liu, C. D. Li, T. W. Huang, and C. J. Li, "Stability of Hopfield neural networks with time delays and variable-time impulses," Neural Computing \& Applications, vol. 22, no. 1, pp. 195-202, 2013.

[22] A. Berman and R. J. Plemmons, Nonnegative Matrices in Mathematical Sciences, Academic Press, New York, NY, USA, 1979.

[23] X. L. Fu, B. Q. Wang, and Y. S. Liu, Nonlinear Impulsive Differential Systems, Science Press, 2008.

[24] X. Liu and G. Ballinger, "Existence and continuability of solutions for differential equations with delays and state-dependent impulses," Nonlinear Analysis, vol. 51, no. 4, pp. 633-647, 2002. 


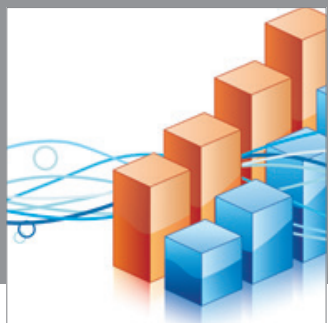

Advances in

Operations Research

mansans

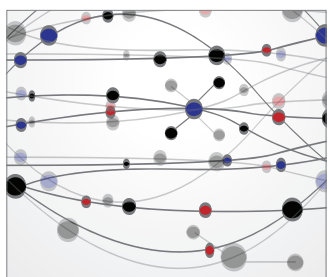

The Scientific World Journal
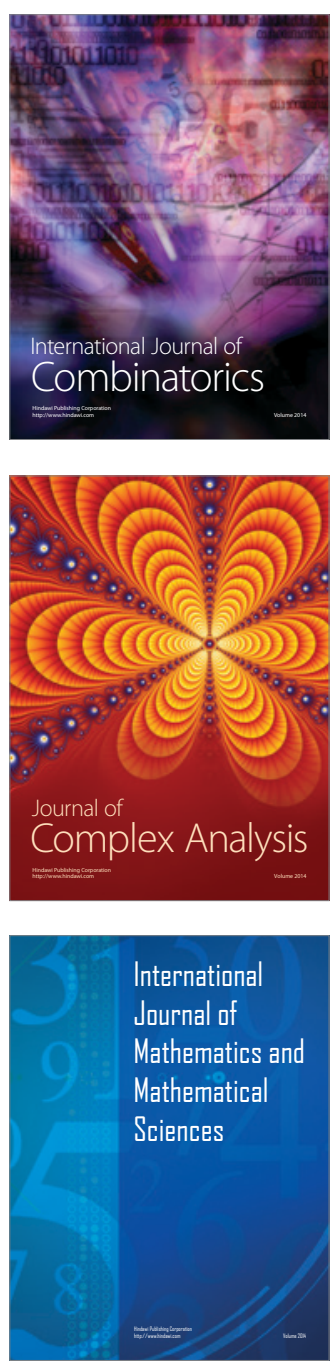
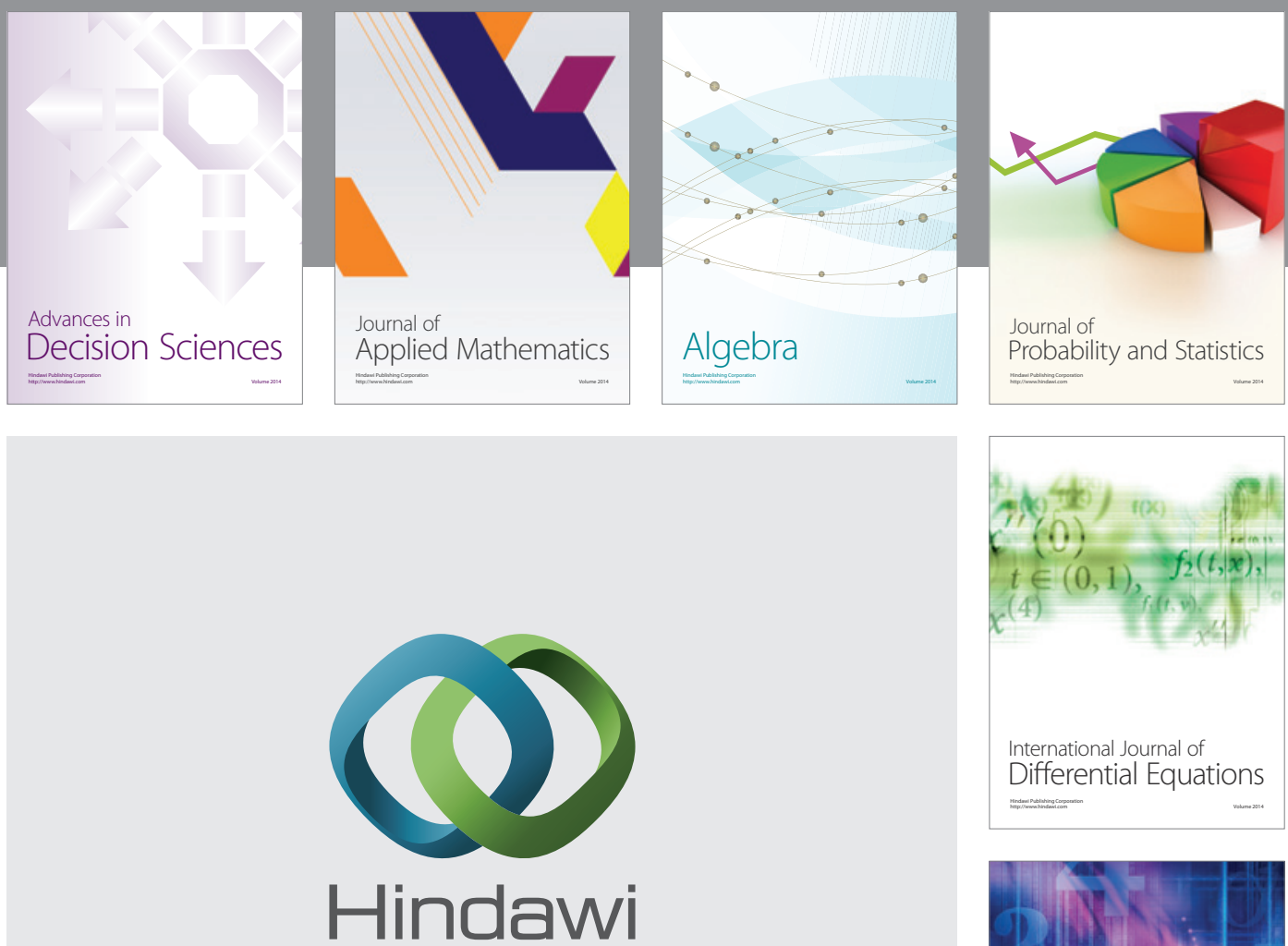

Submit your manuscripts at http://www.hindawi.com
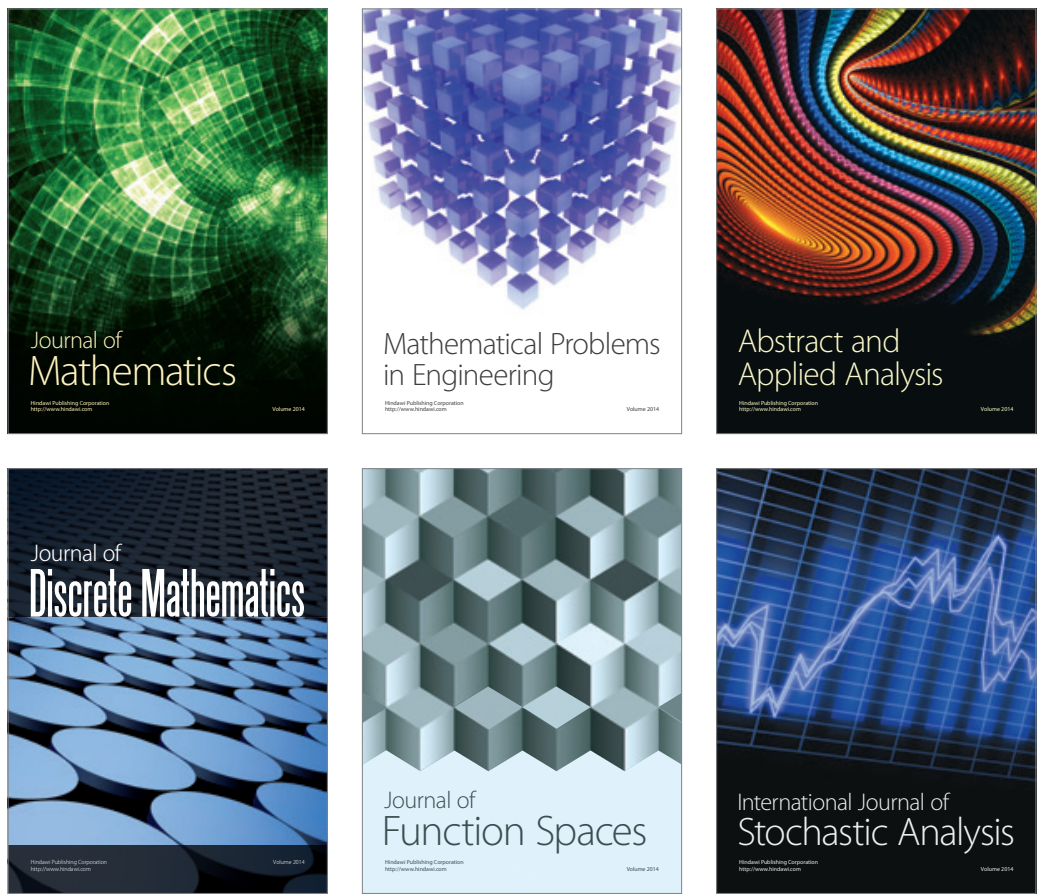

Journal of

Function Spaces

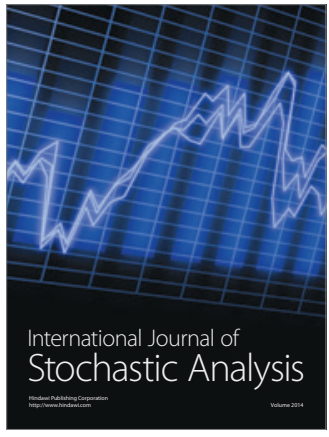

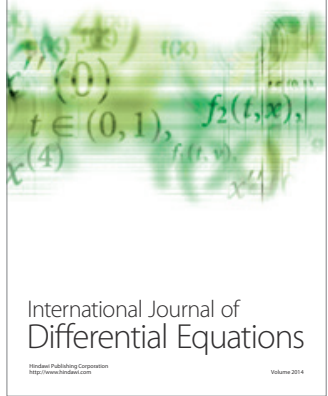
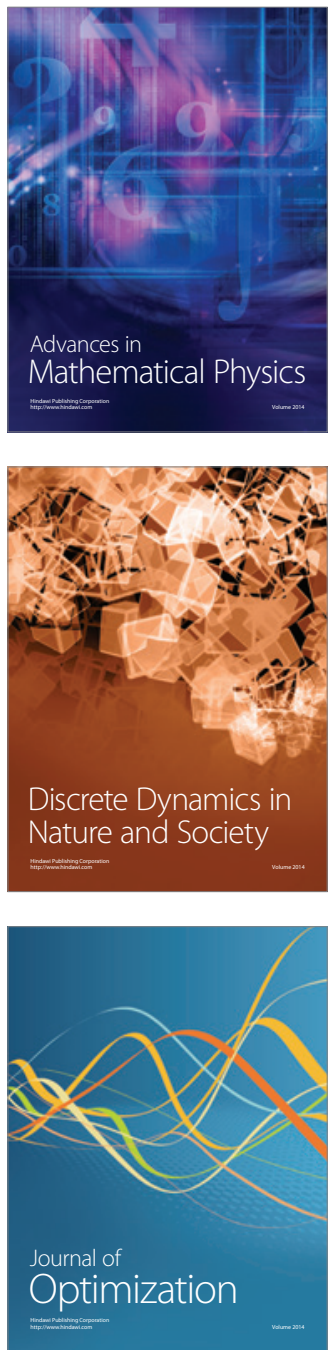